\title{
Psoriasisnägel brauchen Motivation und Geduld
}

\author{
Nagelveränderungen werden von den Betroffenen oft nicht nur als optische Zumutung empfunden, \\ sie können auch verschiedene Tätigkeiten im Alltag einschränken. Vor allem im Zusammenhang \\ mit einer Schuppenflechte kommt es immer wieder zu Problemen mit den Nägeln.
}

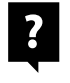

Prof. Dr. med. Knut Schäkel Leitender Oberarzt Universitäts-Hautklinik Heidelberg

\section{? Welche Therapiemöglichkeiten gibt} es bei Nagel-Psoriasis?

Schäkel: Ölflecken sind ja schon Ausdruck einer Entzündung und bei Tüpfelnägeln läuft etwas bei der Verhornung falsch. Deshalb würde ich bereits diese Veränderungen mit wirkstoffhaltigen Präparaten behandeln. Zur örtlichen Therapie verwenden wir entweder antientzündliche Kortisonpräparate oder Vitamin-D3-Analoga, die das starke Wachstum der hornbildenden Zellen hemmen. Bei stärkeren Veränderungen muss der Hautarzt ausschließen, dass sich nicht noch ein Haut- oder Nagelpilz aufgepropft hat. Dicke, aufgeworfene Nagelplatten lassen sich mit harnstoffhaltigen Nagellacken glätten. Auch Ab-

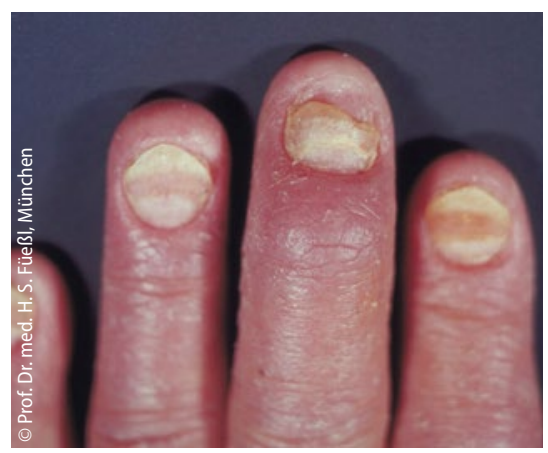

Psoriasis-Nägel können zur therapeutischen Herausforderung werden. fräsen ist bei verformten Nägeln eine Möglichkeit. Innerliche Therapien mit Wirkstoffen wie Biologika wirken sehr gut auch gegen Nagelpsoriasis. Sie werden allgemein eingesetzt, wenn alle anderen Therapien über längere Zeit nicht erfolgreich waren. In Einzelfällen erhalten sie auch Patienten, die noch keine starken Hauterscheinungen haben, aber sehr unter ihren Nagelveränderungen leiden.

\section{? Auf welche Therapiedauer müssen sich die Patienten einstellen?}

Schäkel: Man kommt nur schlecht durch den Nagel hindurch an den Zielort der Behandlung heran. Deshalb ist die Therapie sehr langwierig, hat dann aber durchaus Aussicht auf Erfolg. Die Therapiedauer hängt auch von der Art der Veränderung $\mathrm{ab}$. Wenn eine Entzündung blockiert werden soll, vergehen sicherlich drei Monate, um einen Effekt zu sehen. Die Behandlung einer verdickten Nagelplatte mit harnstoffhaltigen Präparaten dagegen funktioniert schon innerhalb von vier Wochen. Für die grundlegende Veränderung eines Nagels jedoch benötigt eine Behandlung, unabhängig von ihrer Art, einige Monate bis zu einem halben Jahr, weil der Nagel einfach erst herauswachsen muss.

\section{? Wie können die Patienten selbst die Behandlung unterstützen?}

Schäkel: Das Wichtigste ist, dass die Patienten motiviert sind. Denn sie müssen die Therapie konsequent über lange Zeit durchführen. Grundsätzlich sollte man die Nägel gut pflegen, eincremen, abtrocknen und nicht beschädigen. Größe- re Nägel trägt besser der Podologe ab. Wenn sich ein Nagel gelöst hat, muss man aufpassen, dass sich keine feuchten Kammern bilden. Um Infektionen und weitere Komplikationen zu vermeiden, kann es dann sinnvoll sein, auch mal mit Handschuhen zu arbeiten. Ist ein Nagel abgelöst, kann das Nagelbett mit Vitamin-D-3-Analoga und eventuell Kortison gepflegt werden. Je konsequenter dies geschieht, während der neue und gesunde Nagel nachwächst, umso seltener kommt es zu Einrissen oder Blutungen.

Interview: Dr. Christine Starostzik

\section{Tipps bei Nagel-Psoriasis}

- Provokationen wie aggressive Maniküre und Manipulationen am Nagelhäutchen vermeiden

- Nägel kurz schneiden, vor allem, wenn die Hände stark beansprucht sind.

- Vor dem Schneiden die Nägel waschen und einfetten, damit sie weniger splittern.

- Brüchige Nägel erst nach einem lauwarmen Öl- oder Teerbad schneiden.

- Querrillen und verdickte Nägel von erfahrenen medizinischen Fußpflegern abfräsen lassen.

- Leichte, weite Schuhe tragen, die nicht auf die betroffenen, verformten Fußnägel drücken.

- Eine Möglichkeit der optischen Verschönerung sind künstliche Fingernägel.

- Psoriatische Krümelnägel durch eine Okklusivbehandlung (mit entsprechendem Pflaster unter Sauerstoffausschluss) mit 40\% Harnstoff-Paste für 10-14 Tage aufweichen und von einer Fachkraft abfräsen lassen.

Quelle: Altmeyer P. Enzyklopädie der Dermatologie, Venerologie und Umweltmedizin. Springer-Verlag Berlin Heidelberg; online 2016 